\title{
Methotrexate induced encephalopathy in acute lymphoblastic leukemia in Omani children
}

\begin{abstract}
Objectives: To investigate the incidence and clinical profile of methotrexate related encephalopathy in Omani children with pre-B Acute Lymphoblastic Leukemia (ALL).

Methods: A retrospective cohort study of 8 children with Pre B Acute Lymphoblastic Leukemia who developed encephalopathy while being treated according to United Kingdome Research Council (UK MRC) ALL protocol during the period between 2005-2016. Demographic data, clinical presentation, diagnosis and treatment are discussed.

Results: Eight children (4males, 4 females), 7 of them were above 10 years of age, with ALL treated according to intermediate risk UK MRC ALL protocol developed transient encephalopathy during treatment. Six children had one episode, one had two episodes, and last one had 3 episodes. All except one recovered completely within 24-96hours after symptomatic treatment. This one had extensive neurological involvement and took seven days to recover. All children had normal coagulation and thrombophilia studies. Four out of the 8 patients subsequently received intrathecal (IT) cytarabine instead of methotrexate without any neurotoxicity. The other 4 patients received further IT methotrexate. Three of these four had recurrence on re-challenge, while the fourth remained asymptomatic. Seven children with neurotoxicity had MRI findings of white matter changes. Six out of eight children are in complete remission while two died later following relapse.
\end{abstract}

Conclusion: Methotrexate induced encephalopathy can occur after intrathecal methotrexate treatment especially in children older than 10 years of age. Diffusion weighted MRI is the currently available best diagnostic tool. Treatment of methotrexate neurotoxicity is mainly supportive and recovery is usually complete. Further studies are needed to understand the mechanism of neurotoxicity, risk factors, and to define the safe dose to prevent such neurotoxicity.

Keywords: methotrexate, encephalopathy, lymphoblastic leukemia, intrathecal therapy, magnetic resonance imaging
Volume 5 Issue 6 - 2017

\author{
Mathew Zachariah,' Hanan F Nazir, ${ }^{1,2}$ Yasser \\ Wali',2 \\ 'Department of Child Health, Sultan Qaboos University \\ Hospital, Oman \\ ${ }^{2}$ Department of Pediatrics, Alexandria Faculty of Medicine, Egypt
}

Correspondence: Hanan F Nazir, Child Health Department, Sultan Qaboos University Hospital, Muscat, Oman,

E-mail:dr.hanannazir@yahoo.com

Received: October 30, 2017 | Published: December 27, 2017
Abbreviations: ALL, acute lymphoblastic leukemia; IT, intrathecal; MTX, methotrexate; IV, intravenous; IT, intrathecal; CSF, cerebrospinal fluid; CNS, central nervous system; THF, tetrahydrofolate; DWI, diffusion weighted imaging

\section{Introduction}

Survival rates for children with leukemia have increased dramatically over the past few decades. Development of new chemotherapeutic agents and expanded use of older agents have had a major impact on outcome. Methotrexate (MTX) was one of the first drugs discovered to have significant activity against leukemia and still continues as a major component of treatment for acute lymphoblastic leukemia (ALL). ${ }^{1}$ MTX is used as oral, intravenous (IV) or intrathecal (IT) preparations. High dose IV MTX (HDMTX) with leucovorin rescue is also used in some of the ALL cases and may be used as an alternative to radiotherapy. ${ }^{2}$ Toxic effects of MTX include mucositis, hepatotoxicity, nephrotoxicity, myelosuppression and neurotoxicity with acute or chronic encephalopathy., ${ }^{3,4}$ The incidence of acute MTX neurotoxicity is reported as $0.8-15 \%$ depending upon the cumulative dose of MTX, route of administration, age of the patient and amount of leucovorin used. ${ }^{5,6}$ Chronic encephalopathy develops slowly, and may impair neurologic function.
In the current report, we present a series of eight children with ALL who developed acute encephalopathy following intrathecal MTX during consolidation phase/ delayed intensification phase of the chemotherapy protocol. All cases had complete neurological recovery within a week and long term follow up showed no residual neurological deficit.

\section{Methods}

We performed a retrospective cohort study of eight children with ALL, diagnosed and treated at pediatric hematology unit of Sultan Qaboos University Hospital (SQUH), Muscat, Oman. All these children developed encephalopathy after intrathecal MTX administration between January 2005 and December 2016. All the children were stratified as intermediate risk patients at the start of treatment with UK Medical Research Council (MRC) ALL protocol. From January 2005 to December 2015 there were 166 cases of ALL, treated with this protocol at our institution. This protocol consists of a four chemotherapeutic drug induction phase which includes Vincristine, Asparaginase, IT MTX, Daunorubicin and dexamethasone. Five weeks Induction phase is followed by three weeks consolidation phase with weekly IT MTX, cyclophosphamide, low dose cytarabine and asparaginase. Dose of IT MTX used in this 
protocol was $12 \mathrm{mg}$ weekly for three weeks during induction and 3 doses during consolidation phase. Conventional CT and MRI were used to evaluate the neurological symptoms.

Medical records were reviewed for demographic and clinical features, number of IT MTX doses used, time interval for development of encephalopathy, number of episodes, treatment used, recurrence, time taken for complete recovery and MRI brain findings. Diagnosis of MTX encephalopathy was made on the clinical features and MRI brain findings. All children were evaluated and followed up by the same pediatric neurologist. Ethical approval for the study was obtained from the University Medical Research and Ethics Committee (MREC*581)

\section{Results}

During the study period, 8 children (4male, 4female) out of 166 Pre B ALL (4.82\%) developed acute encephalopathy following IT
MTX. The demographic and clinical profile of children is given in Table 1. All affected children were Omanis with an average age of 10.5years (age ranged from 7-12years). All of them had Pre B ALL and were treated with MRC ALL UK protocol. Only one child had CNS involvement with leukemia at presentation. Average number of doses of IT MTX received prior to development of encephalopathy was 6.87 (range 3-13 doses). None of the affected children received high dose MTX. All of them developed encephalopathy within 10 days of receiving IT MTX. The child having CNS involvement with ALL received additional cranial irradiation during $12^{\text {th }}$ to $15^{\text {th }}$ week of treatment. He developed encephalopathy at week $15^{\text {th }}$ of treatment. He had received a total of 10 IT MTX prior to developing encephalopathy and had a recurrence on further Methotrexate therapy. Child number 5 developed encephalopathy at week 33 ; he had received a total of 13 IT MTX prior to encephalopathy. All children had normal coagulation profile, thrombophilia work up and clear cerebrospinal fluid (CSF) at the time of encephalopathy.

Table I Demographic and clinical profile of patients with MTX- induced encephalopathy

\begin{tabular}{|c|c|c|c|c|c|c|c|c|c|c|c|c|c|}
\hline No & $\begin{array}{l}\text { Age } \\
\text { (Yrs) }\end{array}$ & Sex & Diagnosis & $\begin{array}{c}\text { Prior CNS } \\
\text { Involvement }\end{array}$ & $\begin{array}{l}\text { No of } \\
\text { preceding } \\
\text { IT MTX } \\
\text { Doses }\end{array}$ & $\begin{array}{l}\text { High } \\
\text { Dose } \\
\text { MTX }\end{array}$ & $\begin{array}{l}\text { Radio- } \\
\text { therapy }\end{array}$ & $\begin{array}{l}\text { Onset } \\
\text { (Days } \\
\text { after } \\
\text { MTX) }\end{array}$ & Episodes & $\begin{array}{c}\text { Time to } \\
\text { Recovery } \\
\text { (Days) }\end{array}$ & Treatment & $\begin{array}{c}\text { Re-challenge } \\
\text { with Further } \\
\text { MTX }\end{array}$ & Recurrence \\
\hline 1 & 11 & $\mathrm{~F}$ & Pre B ALL & Nil & 3 & Nil & Nil & 5 & 3 & 4 & Dexa & yes & yes \\
\hline 2 & 12 & $\mathrm{~F}$ & Pre BALL & Nil & 5 & Nil & Nil & 10 & 1 & 1 & Nil & No & No \\
\hline 3 & 12 & $\mathrm{~F}$ & Pre BALL & Nil & 4 & Nil & Nil & 5 & 2 & 3 & Dexa & Yes & Yes \\
\hline 4 & 10 & $M$ & Pre B ALL & Nil & 5 & $\mathrm{Nil}$ & Nil & 9 & I & I & Nil & No & No \\
\hline 5 & 12 & $M$ & Pre BALL & Nil & 13 & Nil & Nil & 4 & 1 & 1 & Dexa & No & No \\
\hline 6 & 7 & $M$ & Pre BALL & Yes & 10 & Nil & Yes & 2 & 1 & 2 & Dexa & Yes & Yes \\
\hline 7 & 11 & $M$ & Pre BALL & Nil & 9 & $\mathrm{Nil}$ & $\mathrm{Nil}$ & 10 & I & 3 & Dexa & No & No \\
\hline 8 & 7 & $\mathrm{~F}$ & Pre B ALL & Nil & 6 & $\mathrm{Nil}$ & Nil & 6 & 1 & 7 & Dexa & Yes & No \\
\hline
\end{tabular}

Pre B ALL, precursor B acute lymphoblastic leukemia; IT, intrathecal; MTX, methotrexate; Dexa, dexamethasone

Clinical symptoms and findings on MRI brain during the encephalopathy episode are summarized in Table 2. Initial neurological symptoms included headache, nausea and vomiting. All children had stroke like features in the form of rapid onset hemi paresis. Symptoms and signs progressed over a period of few hours. Four children had

Table 2 Clinical symptoms and findings on MRI of the brain expressive dysphasia and three of them had dysarthria, with intact auditory functions. Five children developed emotional instability like anxiety, inappropriate laughter and unprovoked crying. Three had choreo-athetoid movements. Three children had seizures and transient ataxia.

\begin{tabular}{|c|c|c|c|c|c|c|c|c|c|c|c|}
\hline No & Headache & Weakness & $\begin{array}{l}\text { Hemi } \\
\text { Paresis }\end{array}$ & Seizures & Dysphasia & Dysarthria & $\begin{array}{l}\text { Emotional } \\
\text { Instability }\end{array}$ & $\begin{array}{l}\text { CSF (At the } \\
\text { Time of En- } \\
\text { cephalopathy) }\end{array}$ & $\begin{array}{l}\text { Coagulation/ } \\
\text { Thrombophilia } \\
\text { Work up }\end{array}$ & MRI findings & $\begin{array}{l}\text { Patient } \\
\text { Condition }\end{array}$ \\
\hline I & Yes & Yes & Yes & No & Yes & Yes & Yes & Normal & Normal & $\begin{array}{l}\text { Hyperdensity in } \\
\text { frontal and Parietal } \\
\text { lobes White Matter }\end{array}$ & $\begin{array}{l}\text { In complete } \\
\text { remission } \\
\text { Normal > } 5 \\
\text { years }\end{array}$ \\
\hline 2 & Yes & Yes & Yes & No & Yes & Yes & No & Normal & Normal & $\begin{array}{l}\text { Hyperintensity in } \\
\text { frontal lobes White } \\
\text { Matter }\end{array}$ & $\begin{array}{l}\text { In complete } \\
\text { remission } \\
\text { Normal }>5 \\
\text { years }\end{array}$ \\
\hline 3 & Yes & Yes & Yes & No & Yes & Yes & Yes & Normal & Normal & $\begin{array}{l}\text { Not done due to } \\
\text { technical reasons }\end{array}$ & $\begin{array}{l}\text { In complete } \\
\text { remission } \\
\text { Normal>5 } \\
\text { years }\end{array}$ \\
\hline
\end{tabular}


Table Continued....

\begin{tabular}{|c|c|c|c|c|c|c|c|c|c|c|c|}
\hline No & Headache & Weakness & $\begin{array}{l}\text { Hemi } \\
\text { Paresis }\end{array}$ & Seizures & Dysphasia & Dysarthria & $\begin{array}{l}\text { Emotional } \\
\text { Instability }\end{array}$ & $\begin{array}{l}\text { CSF (At the } \\
\text { Time of En- } \\
\text { cephalopathy) }\end{array}$ & $\begin{array}{l}\text { Coagulation/ } \\
\text { Thrombophilia } \\
\text { Work up }\end{array}$ & MRI findings & $\begin{array}{l}\text { Patient } \\
\text { Condition }\end{array}$ \\
\hline 4 & No & Yes & Yes & Yes & No & No & No & Normal & Normal & $\begin{array}{l}\text { Focal areas of } \\
\text { demyelinization at } \\
\text { angles of ventricles } \\
\text { and hyperintensity } \\
\text { of periventricular } \\
\text { whiter matter. }\end{array}$ & $\begin{array}{l}\text { In Complete } \\
\text { Remission } \\
\text { Normal }>5 \\
\text { Years }\end{array}$ \\
\hline 5 & Yes & Yes & Yes & Yes & Yes & No & No & Normal & Normal & $\begin{array}{l}\text { Multiple nodular } \\
\text { deep white matter } \\
\text { enhancing lesion. }\end{array}$ & $\begin{array}{l}\text { In complete } \\
\text { remission } \\
\text { Normal > } 5 \\
\text { years }\end{array}$ \\
\hline 6 & Yes & Yes & Yes & Yes & No & No & Yes & Normal & Normal & $\begin{array}{l}\text { Diffuse white } \\
\text { matter changes } \\
\text { in subcortical } \\
\text { white matter } \\
\text { in both frontal, } \\
\text { frontoparietal and } \\
\text { parieto occipital } \\
\text { region. }\end{array}$ & $\begin{array}{l}\text { Died after } \\
\text { relapse }\end{array}$ \\
\hline 7 & Yes & Yes & Yes & No & No & No & Yes & Normal & Normal & $\begin{array}{l}\text { Enhancing lesion } \\
\text { at periventricular, } \\
\text { thalamus and } \\
\text { Midbrain area }\end{array}$ & $\begin{array}{l}\text { Died after } \\
\text { relapse }\end{array}$ \\
\hline 8 & Yes & Yes & Yes & No & No & No & Yes & Normal & Normal & $\begin{array}{l}\text { Hyperintensity in } \\
\text { frontal lobes White } \\
\text { Matter }\end{array}$ & $\begin{array}{l}\text { Alive Post } \\
\text { BMT }\end{array}$ \\
\hline
\end{tabular}

All children had conventional CT scan of the brain soon after the onset of neurological symptoms. All CT scans were normal except one showed multiple focal petechial hemorrhages. Seven children had MRI brain within two days of onset of encephalopathy. One child did not have MRI due to technical reasons. MRI findings varied from atrophy, focal white matter changes to diffuse white matter changes. Findings are shown in Table 2 and Figure 1- 4.

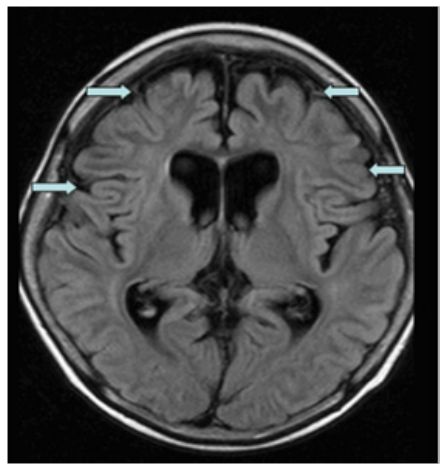

Figure I Axial view of the MRI brain (FLAIR) showing diffuse atrophy of the brain.

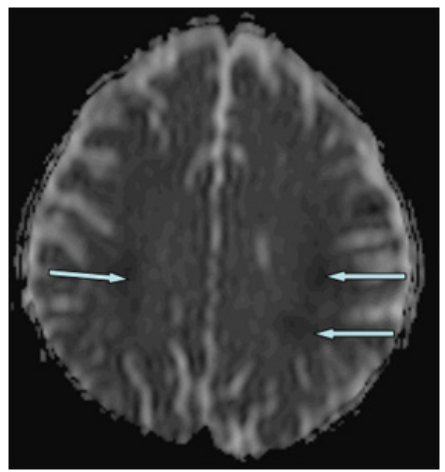

Figure 2 Axial view diffusion weighted image on MRI showing areas of hypointense lesions.

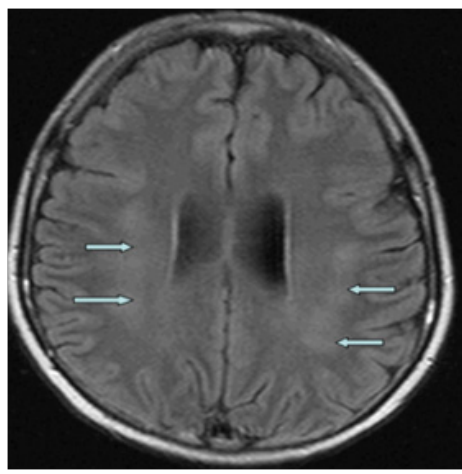

Figure 3 Axial view MRI (FLAIR) showing multiple focal hyperintense lesions scattered in the white matter.

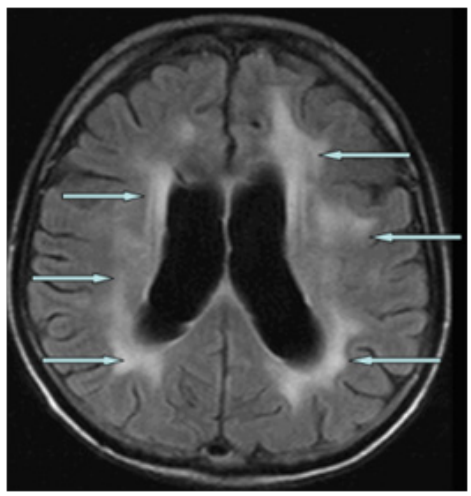

Figure 4 Axial view MRI (FLAIR) showing diffuse periventricular white matter changes.

All children were treated with dexamethasone and phenytoin was added to those with seizures. After recovery from encephalopathy, IT Cytarabine was used instead of IT MTX in 4 children for completion of chemotherapy. Four out of the eight received further methotrexate. Three of them had neurologic manifestations upon re-challenge while the fourth remained asymptomatic. 


\section{Discussion}

The current report focus on encephalopathy as one of the uncommon neurotoxic manifestations of intrathecal methotrexate administration in pediatric patients with ALL. Central nervous system (CNS) prophylaxis by cranial irradiation or intrathecal/high dose MTX are important in the prevention of CNS relapse in childhood ALL. MTX can be used as an effective alternative to radiotherapy in children. However, MTX has a significant toxic effect on CNS, and can cause acute, sub acute or chronic neurotoxity. ${ }^{7}$ In the current cohort, incidence of methotrexate related encephalopathy was $4.8 \%$, and all cases developed neurotoxicity in relation to IT MTX. Comparable incidence was reported by Bhojwani et al., ${ }^{8}$ who reported $3.8 \%$ clinical neurotoxicity, but mostly related to high dose IV MTX. ${ }^{8}$ Clinical presentation of MTX related neurotoxicity is highly variable, ranging from asymptomatic (diagnosed on imaging) to seizures, coma and death. ${ }^{9}$ In our cohort, the most common manifestations were weakness and hemiparesis occurring in $100 \%$ of cases. Recently, several authors reported on MTX related neurotoxicity presenting with stroke-like clinical picture. ${ }^{10-12}$

Common manifestations reported include headache, dizziness, tremor, ataxia, aphasia, dysarthria, emotional instabilities, seizures, hemiparesis and leukoencephalopathy. ${ }^{13,14}$ Acute encephalopathy usually resolves within a week, but may recur after MTX therapy is resumed. In our study group, most patients recovered within 7 days. MTX was resumed in four children; three of them had recurrence of neurotoxicity upon re-challenge. Onset of encephalopathy varies, two children developed acute encephalopathy late in the course of the treatment. One of them at $15^{\text {th }}$ week after receiving cranial radiotherapy at $12-15^{\text {th }}$ weeks. This encephalopathy might not be related to radiation as there was recurrence when IT MTX was resumed alone later. Other child had encephalopathy at 33weeks of therapy. Neurological symptoms like somnolence, confusion, fatigue and seizures have been reported to occur after high dose MTX. ${ }^{15}$ However, in our children they only occurred with IT MTX (Table 2). Myelopathy and symptoms like leg pain, sensory changes and bladder dysfunction are also reported with IT MTX. ${ }^{16}$ These were not seen in our children. It worth mentioning that none of our T Cell Leukemic children who received high doses of MTX developed any neurotoxicity.

Age $\geq 10 y e a r$ is associated with an increased incidence of MTX neurotoxicity; the reasons for this remain unexplained. ${ }^{17}$ Consistent with this observation, seven of our patients were older than 10years. Combination of other chemotherapeutic agents and radiotherapy with MTX increases the risk of developing neurotoxicity. Disruption of the blood brain barrier by radiotherapy increases the penetration of MTX to the brain. Only one of our patients had IT MTX combined with radiotherapy. The radiotherapy could explain the first encephalopathy in that child but not the recurrence on re-challenge, when IT MTX was used alone. The mechanism of acute MTX-induced neurotoxicity remains unclear. One possible mechanism is that MTX inhibits enzyme dihydrofolate reductase, necessary for reduction of dihydrofolate to tetrahydrofolate (THF). Deficiency of THF leads to depletion of intracellular folates and result in decreased synthesis of purines which prolong the intracellular retention of MTX leading to increased adenosine levels in the CSF. High adenosine levels can dilate cerebral blood vessels, modify the release of pre and post synaptic neurotransmitters and may slow the discharge rate of neurons. Hence adenosine release may contribute to the pathophysiology of acute MTX neurotoxicity. ${ }^{9}$
Diagnosis of MTX neurotoxicity is made from the clinical presentation and imaging studies. CT scans and T1 or T2 weighted MRI and angiography does not show consistent abnormalities that characterize MTX induced neurotoxicity. Diffusion weighted imaging (DWI) appears to be more sensitive in the diagnosis. Diagnosis can be confirmed when DWI shows areas of restricted diffusion across multiple vascular beds and involvement of deep cerebral white matter, in presence of clinical signs and symptoms. ${ }^{18,19}$ FLAIR images help to differentiate the demyelinating lesion from inflammatory one. Treatment of MTX neurotoxicity is often using aminophylline, which is a competitive antagonist of adenosine, but the clinical efficacy remains controversial at the moment. ${ }^{14}$ Currently, the treatment for methotrexate induced encephalopathy remains mainly supportive eg: removal of MTX from treatment protocol, use of steroids to reduce white matter edema, use of folinic acid, and anticonvulsive drugs if there are seizures. ${ }^{9}$ Most children with encephalopathy have a favorable outcome and the neurological deficits are completely reversible. However, long term neurological effects remain unknown in terms of cognitive function. All our children had complete recovery within two weeks; follow up so far did not show any neurological deficit or intellectual impairment. Two of our children died of disease relapse, and the other six are in remission.

\section{Conclusion}

Methotrexate induced encephalopathy can occur after intrathecal methotrexate treatment especially in children older than 10 years of age. Diffusion weighted MRI is the currently available best diagnostic tool. Treatment of methotrexate neurotoxicity is mainly supportive and recovery is usually complete. Further studies are needed to understand the mechanism of neurotoxicity, risk factors, and find the safe dose to prevent such neurotoxicity.

\section{Acknowledgements}

None.

\section{Conflict of interest}

The author declares no conflict of interest.

\section{References}

1. Farber S. Some observations on the effect of folic acid antagonists on acute leukemia and other forms of incurable cancer. Blood. 1949;4(2):160-167.

2. Pui CH, Evans WE. Treatment of acute lymphoblastic leukemia. $N$ Engl J Med. 2006;354(2):166-178.

3. Atra A, Pinkerton CR, Bouffet $\mathrm{E}$, et al. Acute neurotoxicity in children with advanced stage B-non-Hodgkin's lymphoma and B-acute lymphoblastic leukaemia treated with the United Kingdom children cancer study group 9002/9003 protocols. Eur J Cancer. 2004;40(9):1346-1350.

4. Shuper A, Stark B, Kornreich L, et al. Methotrexate treatment protocols and the central nervous system:significant cure with significant neurotoxicity. J Child Neurol. 2000;15(9):573-580.

5. Rubnitz JE, Relling MV, Harrison PL, et al. Transient encephalopathy following high-dose Methotrexate treatment in Childhood acute lymphoblastic leukemia. Leukemia. 1998;12(8):1176-1181.

6. Dufourg MN, Landman-Parker J, et al. Age and high dose methotrxate are associated to clinical acute encephalopathy in FRALLE 93 trial for acute lymphoblastic leukemia in children. Leukemia. 2007;21(2):238247. 
7. Vezmar S, Schüsseler P, Becker A, et al. Methotrxate-associated alterations of the folate and methyl-transfer pathway in the CSF of ALL patients with and without symptoms of neurotoxicity. Pediatr Blood Cancer. 2009;52(1):26-32.

8. Bhojwani D, Sabin ND, Pei D, et al. Methotrexate-induced neurotoxicity and leukoencephalopathy in childhood acute lymphoblastic leukemia. $J$ Clin Oncol. 2014;32(9):949-959.

9. Aradillas E, Arora R, Gasperino J. Methotrexate induced posterior reversible encephalopathy sundrome. $J$ Clin Pharm Ther. 2011;36(4):529-536.

10. Cruz-Carreras MT, Chaftari P, Shamsnia A, et al. Methotrexate-induced leukoencephalopathy presenting as stroke in the emergency department. Clin Case Rep. 2017;5(10):1644-1648.

11. Gosavi TD, Ahmad MT, Lee LH, et al. Methotrexate induced leucoencephalopathy: A stroke mimic. Ann Indian Acad Neurol. 20113;16(3):418-421.

12. Agarwal A, Vijay K, Thamburaj K, et al. Transient leukoencephalopathy after intrathecal methotrexate mimicking stroke. Emerg Radiol. 2011;18(4):345-347.

13. Shuper A, Stark B, Kornreich L, et al. Methotrexate-Related neurotoxicity in the treatment of childhood Acute lymphoblastic leukemia. Isr Med Assoc J. 2002;4(11):1050-1053.
14. LAM DST, KHONG PL, CHIANGAKS, et al. Reversible encephalopathy induced by systemic high dose Methotrexate. HKJ Paediatr (new series). 2007;12:287-292.

15. Walker RW, Allen JC, Rosen G, et al. Transient cerebral dysfunction secondary to high dose methotrexate. J Clin Oncol. 1986;4(12):18451850 .

16. Yim YS, Mahoney DH, Oshman DG. Hemiparesis and ischemic changes of the white matter after intrathecal therapy for children with acute lymphocytic leukemia. Cancer. 1991;67(8):2058-2061.

17. Rubnitz JE, Relling MV, Harrison PL, et al. Transient encephalopathy following high dose methotrexate treatment in childhood acute lymphoblastic leukemia. Leukemia. 1998;12(8):1176-1181.

18. Rollins N, Winick N, Bash R, et al. Acute methotrexate neurotoxicity: findings on diffusion-weighted imaging and correlation with clinical outcome. AJNR Am J Neuroradiol. 2004;25(10):1688-1695.

19. Haykin ME, Gorman M, van Hoff J, et al. Diffusion-weighted MRI correlates of sub-acute methotrexate related neurotoxicity. $J$ Neurooncol. 2006;76(2):153-157. 